\title{
Tumor Marker Evolution: Comparison with Imaging for Assessment of Response to Chemotherapy in Patients with Colorectal Liver Metastases
}

\author{
Robbert J. de Haas, MD ${ }^{1,2}$, Dennis A. Wicherts, $\mathrm{MD}^{1,2}$, Eduardo Flores, $\mathrm{MD}^{1}$, Michel Ducreux, MD, $\mathrm{PhD}^{3}$, \\ Francis Lévi, MD, $\mathrm{PhD}^{3,4}$, Bernard Paule, $\mathrm{MD}^{3}$, Daniel Azoulay, $\mathrm{MD}, \mathrm{PhD}^{1}$, Denis Castaing, $\mathrm{MD}^{1,5,6}$, \\ Antoinette Lemoine, $\mathrm{PhD}^{7}$, and René Adam, $\mathrm{MD}, \mathrm{PhD}^{1,5,6}$ \\ ${ }^{1}$ Centre Hépato-Biliaire, AP-HP Hôpital Paul Brousse, Villejuif, France; ${ }^{2}$ Department of Surgery, University Medical \\ Center Utrecht, Utrecht, The Netherlands; ${ }^{3}$ Department of Medical Oncology, AP-HP Hôpital Paul Brousse, Villejuif, \\ France; ${ }^{4}$ Laboratoire 'Rythmes biologiques et cancers', INSERM, Unité 776, Villejuif, France; ${ }^{5}$ Université Paris-Sud, \\ Villejuif, France; ${ }^{6}$ INSERM, Unité 785, Villejuif, France; ${ }^{7}$ Department of Biochemistry, AP-HP Hôpital Paul Brousse, \\ Villejuif, France
}

\begin{abstract}
Background. As the real clinical significance of carcinoembryonic antigen (CEA) and carbohydrate antigen 19.9 (CA19.9) evolution during preoperative chemotherapy for colorectal liver metastases (CLM) is still unknown, we explored the correlation between biological and radiological response to chemotherapy, and their comparative impact on outcome after hepatectomy.

Methods. All patients resected for CLM at our hospital between 1990 and 2004 with the following eligibility criteria were included in the study: (1) preoperative chemotherapy, (2) complete resection of CLM, (3) no extrahepatic disease, and (4) elevated baseline tumor marker values. A $20 \%$ change of tumor marker levels while on chemotherapy was used to define biological response (decrease) or progression (increase). Correlation between biological and radiological response at computed tomography (CT) scan, and their impact on overall survival (OS) and progression-free survival (PFS) after hepatectomy were determined.

Results. Among 119 of 695 consecutive patients resected for CLM who fulfilled the inclusion criteria, serial CEA and CA19.9 were available in 113 and 68 patients, respectively. Of patients with radiological response or stabilization, $94 \%$
\end{abstract}

(C) The Author(s) 2010. This article is published with open access at Springerlink.com

First Received: 16 August 2009;

Published Online: 6 January 2010

R. Adam, MD, PhD

e-mail: rene.adam@pbr.aphp.fr had similar biological evolution for CEA and $91 \%$ for CA19.9. In patients with radiological progression, similar biological evolution was observed in $95 \%$ of cases for CEA and in $64 \%$ for CA19.9. On multivariate analysis, radiological response (but not biological evolution) independently predicted OS. However, progression of CA19.9, but not radiological response, was an independent predictor of PFS. Conclusions. In patients with CLM and elevated tumor markers, biological response is as accurate as CT imaging to assess "clinical" response to chemotherapy. With regards to PFS, CA19.9 evolution has even better prognostic value than does radiological response. Assessment of tumor markers could be sufficient to evaluate chemotherapy response in a nonsurgical setting, limiting the need of repeat imaging.

Hepatic resection is still considered the most effective treatment for patients with colorectal liver metastases (CLM). ${ }^{1}$ Unfortunately, only $10-20 \%$ of patients are directly amenable to surgery. ${ }^{2}$ In the remaining patients, complete metastatic resection would result in a too small volume of remnant functional liver parenchyma. However, due to major improvements in chemotherapy regimens, 13 $54 \%$ of initially unresectable patients can now be switched to resectability. ${ }^{3-9}$ Besides this, systemic chemotherapy is increasingly being used in a neoadjuvant setting (i.e., in initially resectable patients) with the aim of facilitating hepatic resection and improving long-term outcome. ${ }^{10,11}$

To determine the efficacy of preoperative chemotherapy, computed tomography (CT) is the gold standard. 
Imaging studies are necessary to determine resectability. Furthermore, best surgical candidates can be identified, as disease progression while on preoperative chemotherapy is associated with poor outcome. ${ }^{12}$ Besides CT imaging, carcinoembryonic antigen (CEA) and/or carbohydrate antigen 19.9 (CA19.9) are widely used to monitor patients while on chemotherapy. Although the prognostic significance of increased preoperative CEA and CA19.9 levels has been well established in large series, clinical significance of tumor marker evolution regarding correlation with imaging studies and impact on long-term outcome is still unclear. ${ }^{13-17}$ To date, only limited data is available suggesting a correlation between CEA evolution and chemotherapy response at CT imaging in patients with advanced colorectal cancer. ${ }^{18-20}$ As patient selection often was not well defined, data interpretation remains difficult. Recently, the American Society of Clinical Oncology recommended CEA as the marker of choice for monitoring metastatic colorectal cancer during systemic therapy. ${ }^{21}$ On the contrary, insufficient data was available to recommend use of CA19.9 for evaluating treatment results in metastatic colorectal cancer patients. ${ }^{21}$

The aim of the present study is to explore the correlation between biological and radiological response following preoperative chemotherapy, and their comparative impact on long-term outcome, for both CEA and CA19.9, in a well-defined population of patients with isolated CLM, scheduled for hepatectomy.

\section{PATIENTS AND METHODS}

\section{Patients}

All consecutive patients treated for CLM at our hospital between January 1990 and January 2004 with the following eligibility criteria were entered in the study: (1) treated by hepatectomy preceded by chemotherapy, (2) data for at least one tumor marker available, (3) primary tumor resected at least 6 months before last preoperative chemotherapy, (4) no extrahepatic disease diagnosed before or during hepatectomy, (5) CT reports before and after preoperative chemotherapy available, and (6) elevated tumor marker levels at baseline measurement (i.e., before preoperative chemotherapy). Patients were selected from our prospectively collected institutional database, and the medical record of each patient was reviewed.

\section{Preoperative Management}

Before hepatectomy, all patients were treated with at least one line of chemotherapy, either to achieve resectability in patients with initially unresectable CLM (i.e., inability to resect the total amount of CLM while leaving at least $30 \%$ future remnant liver volume) or in a neoadjuvant setting in patients with synchronous (i.e., diagnosed before, during or within 3 months after colorectal resection) or marginally resectable CLM (i.e., multinodular bilateral CLM).

In every patient, serum CEA and/or CA19.9 levels were routinely measured before, during, and after preoperative chemotherapy at our institution. Tumor markers were measured in fresh sera using Architect I2000SR CMIA technology (Abbott Diagnostics, Abbott Park, IL). Tumor marker levels $<5 \mathrm{ng} / \mathrm{ml}$ for CEA and $<37 \mathrm{U} / \mathrm{ml}$ for CA19.9 were considered normal.

Clinical response at CT imaging during chemotherapy was routinely evaluated every 2 months in a multidisciplinary staff meeting, including surgeons, medical oncologists, and radiologists. For every patient, the CT scan was reviewed by an expert radiologist blinded to tumor marker evolution, and type of radiological response after the last preoperative chemotherapy line was determined according to the Response Evaluation Criteria in Solid Tumors (RECIST). ${ }^{22}$

\section{Hepatic Resection}

All patients underwent hepatic resection with curative intent, after exploration by intraoperative ultrasound. If needed to allow resection, preoperative portal vein embolization was performed. ${ }^{23}$ Hepatic resections were classified as major ( $\geq 3$ segments) or limited ( $<3$ segments). ${ }^{24,25}$

\section{Postoperative Follow-Up}

Postoperatively, all patients were followed up regularly, starting 1 month after the operation and then every 4 months, consisting of clinical examination, serum tumor markers, abdominal ultrasound, and thoracoabdominal imaging. To reduce risk of recurrence, adjuvant chemotherapy was routinely recommended. If intra- and/or extrahepatic disease recurrence occurred which could be resected curatively, repeat resection was performed. ${ }^{26}$

\section{Statistical Considerations}

Mean tumor marker levels before and after the last preoperative chemotherapy were compared by pairedsamples $t$ test. To analyze the clinical significance of tumor marker evolution, a cutoff point of $20 \%$ change in tumor marker level after chemotherapy was chosen, as best agreement between radiological and biological response was observed when using this cutoff point, as assessed by the Cohen kappa test. A decrease of tumor marker level of $20 \%$ or more was defined as biological response. Likewise, an increase of tumor marker level of $20 \%$ or more was 
defined as biological progression. A decrease or increase of tumor marker level by less than $20 \%$ was considered as biological stabilization. To analyze the correlation between biological and radiological response after chemotherapy, as well as the impact of tumor marker evolution on survival, response and stabilization were grouped together (positive cases) and compared with progression (negative cases). Sensitivity, specificity, positive predictive value (PPV), and negative predictive value (NPV) of tumor marker evolution were calculated, considering radiological response at $\mathrm{CT}$ imaging as reference standard.

Overall and progression-free survival curves were calculated by Kaplan-Meier method, and survivals of different groups were compared using log-rank tests. Survival rates were calculated from time of hepatectomy. Univariate associations between study variables (including patient, primary tumor, initial CLM, chemotherapy, and hepatectomy characteristics) and both overall and progression-free survival were determined by log-rank test $P$ value $\leq 0.05$. To identify independent predictors of overall and progressionfree survival, factors with univariate $P$ value $\leq 0.10$ were entered in a multivariate Cox proportional hazard model. $P$ value $\leq 0.05$ was considered statistically significant. All statistical analyses were performed by using SPSS version 13.0 (SPSS Inc., Chicago, IL).

\section{RESULTS}

\section{Patient Characteristics}

Between 1990 and 2004, 695 patients underwent hepatic resection for CLM at our hospital. Of these, 119 patients (17\%) fulfilled the inclusion criteria and were subsequently enrolled in the study (Fig. 1). The study population consisted of 79 men (66\%) and 40 women (34\%), and mean age was 61 years (Table 1). In 95 patients $(80 \%)$, the primary tumor was located in the colon. Forty-one patients (34\%) presented with CLM synchronous to the primary colorectal malignancy. It concerned only one liver metastasis in 34 patients (30\%), and metastases were initially unresectable in 55 patients $(46 \%)$.

\section{Preoperative Chemotherapy}

Before hepatic resection, all patients were treated by at least one line of chemotherapy. A median number of 1 line (range 1-5) and a median number of 7 cycles (range 2-33) of systemic chemotherapy were administered. Radiological response of CLM after chemotherapy was observed in 72 patients $(60 \%)$, stabilization in $28(24 \%)$, and disease progression in 19 (16\%). Chemotherapy details are indicated in Table 2.

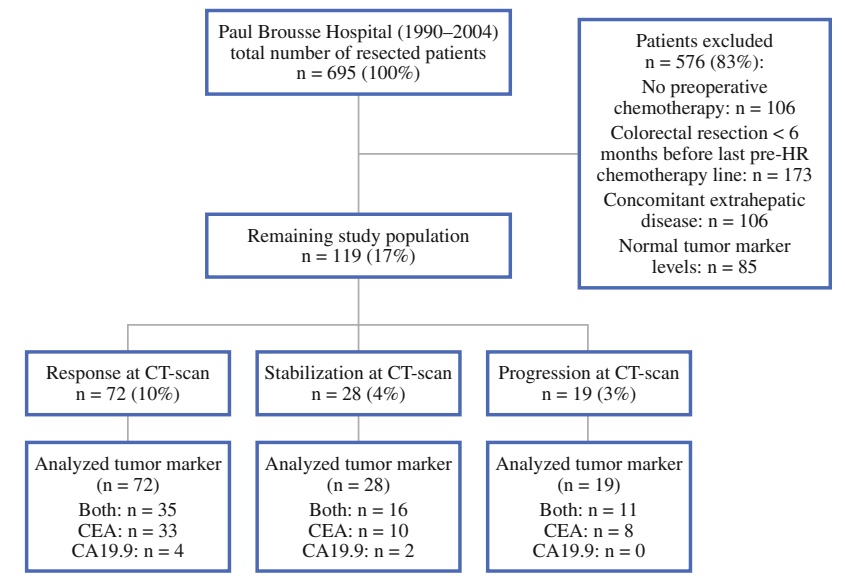

FIG. 1 Flowchart of study population. $H R$ hepatic resection, $C T$ computed tomography, $C E A$ carcinoembryonic antigen, $C A$ carbohydrate antigen

TABLE 1 Patient and tumoral characteristics

\begin{tabular}{ll}
\hline Characteristics & Total study population $(N=119)$ \\
\hline Patients & \\
Mean age at HR, years \pm SD & $61 \pm 10$ \\
Male/female & $79(66 \%) / 40(34 \%)$ \\
Primary tumor & \\
Colon/rectum & $95(80 \%) / 24(20 \%)$ \\
T classification & \\
1 & $2(2 \%)$ \\
2 & $7(8 \%)$ \\
3 & $41(49 \%)$ \\
4 & $33(40 \%)$ \\
N classification & \\
0 & $33(39 \%)$ \\
1 & $28(33 \%)$ \\
2 & $24(28 \%)$
\end{tabular}

Liver metastases at initial diagnosis

$\begin{array}{ll}\text { Synchronous/metachronous }^{\mathrm{a}} & 41(34 \%) / 78(66 \%) \\ \text { Number of CLM } & \\ 1 & 34(30 \%) \\ 2-3 & 44(38 \%) \\ >3 & 37(32 \%) \\ \text { Mean maximum size, mm } \pm \mathrm{SD} & 46 \pm 32 \\ \text { Unilateral/bilateral } & 61(52 \%) / 57(48 \%) \\ \text { Initial unresectability } & 55(46 \%)\end{array}$

$H R$ hepatic resection, $S D$ standard deviation, $C L M$ colorectal liver metastases

${ }^{\text {a }}$ Synchronous $=$ diagnosed before, during, or within 3 months after colorectal resection

\section{Tumor Marker Evolution}

Data to determine the biological response were available in 113 cases for CEA and in 68 cases for CA19.9 
TABLE 2 Operative features

5-FU 5-fluorouracil, $L V$

leucovorin, $C T$ computed

tomography, CEA

carcinoembryonic antigen, $C A$

carbohydrate antigen, $P V E$

portal vein embolization, CLM

colorectal liver metastases, $S D$

standard deviation

a According to RECIST criteria $^{22}$

${ }^{\mathrm{b}}$ Cutoff point $20 \%$

c R0: complete surgical resection with a negative surgical margin at

histopathology; R1: invaded

surgical margins according to

the pathologist; R2:

macroscopic tumor remnant intraoperatively

${ }^{\mathrm{d}}$ Hepatic complications considered were: biliary leak/ bilioma, hemorrhage, infected collection, noninfected collection, and transient liver insufficiency

e General complications considered were: pulmonary, cardiovascular, urinary tract, infectious (other than local hepatic), and iatrogenic complications

\begin{tabular}{|c|c|}
\hline Feature & Total study population $(N=119)$ \\
\hline Preoperative chemotherapy & $119(100 \%)$ \\
\hline \multicolumn{2}{|l|}{ Total no. of lines } \\
\hline 1 & $87(73 \%)$ \\
\hline$>1$ & $32(27 \%)$ \\
\hline \multicolumn{2}{|l|}{ Total no. of cycles } \\
\hline$<6$ & $27(24 \%)$ \\
\hline$\geq 6$ & $85(76 \%)$ \\
\hline \multicolumn{2}{|l|}{ Regimen last preoperative line } \\
\hline 5-FU LV & $32(27 \%)$ \\
\hline 5-FU LV oxaliplatin & $61(51 \%)$ \\
\hline 5-FU LV irinotecan & $18(15 \%)$ \\
\hline Other & $8(7 \%)$ \\
\hline Chronomodulated delivery & $59(50 \%)$ \\
\hline \multicolumn{2}{|l|}{ Clinical response at CT $\operatorname{scan}^{\mathrm{a}}$} \\
\hline Response & $72(60 \%)$ \\
\hline Stabilization & $28(24 \%)$ \\
\hline Progression & $19(16 \%)$ \\
\hline \multicolumn{2}{|l|}{ Change in CEA level after chemotherapy $(N=113)^{\mathrm{b}}$} \\
\hline Response & $75(66 \%)$ \\
\hline Stabilization & $14(12 \%)$ \\
\hline Progression & $24(21 \%)$ \\
\hline \multicolumn{2}{|l|}{ Change in CA19.9 level after chemotherapy $(N=68)^{\mathrm{b}}$} \\
\hline Response & $48(71 \%)$ \\
\hline Stabilization & $8(12 \%)$ \\
\hline Progression & $12(18 \%)$ \\
\hline \multicolumn{2}{|l|}{ Hepatectomy } \\
\hline PVE & $14(12 \%)$ \\
\hline Major hepatectomy ( $\geq 3$ segments) & $62(52 \%)$ \\
\hline Mean no. of CLM at histopathology \pm SD & $3 \pm 3$ \\
\hline Mean maximum size of CLM at histopathology, $\mathrm{mm} \pm \mathrm{SD}$ & $44 \pm 36$ \\
\hline \multicolumn{2}{|l|}{ Surgical margin status ${ }^{c}$} \\
\hline R0 & $71(60 \%)$ \\
\hline $\mathrm{R} 1$ & $43(36 \%)$ \\
\hline $\mathrm{R} 2$ & $4(3 \%)$ \\
\hline Postoperative mortality ( $\leq 2$ months) & $1(1 \%)$ \\
\hline Postoperative complications & 43 patients $(36 \%)$ \\
\hline Hepatic $^{\mathrm{d}}$ & $31(26 \%)$ \\
\hline General $^{\mathrm{e}}$ & $20(17 \%)$ \\
\hline Postoperative chemotherapy & $105(88 \%)$ \\
\hline
\end{tabular}

(Fig. 1). Changes in tumor marker levels after chemotherapy within the total study population are shown in Table 2 .

The mean CEA level after chemotherapy in patients with radiological response of CLM was significantly lower compared with the mean value before preoperative chemotherapy (Fig. 2a). If either stabilization or progression of CLM was observed following chemotherapy, mean CEA level did not significantly differ from that determined before starting preoperative chemotherapy (Fig. 2b, c). When using a cutoff point of $20 \%$ to define biological response, agreement between biological (CEA) and radiological response was observed in $80 \%$ of cases (90/113), with a kappa value of 0.62 [95\% confidence interval (CI): 0.49-0.76] (Table 3). Sensitivity was $94 \%$ (95\% CI: $89-$ $99 \%$ ), specificity was $95 \%$ (95\% CI: 85-105\%), and PPV and NPV were $99 \%$ (95\% CI: $97-101 \%)$ and $75 \%(95 \%$ CI: $58-92 \%)$, respectively. 
a

Mean CEA

Level (ng/ml)

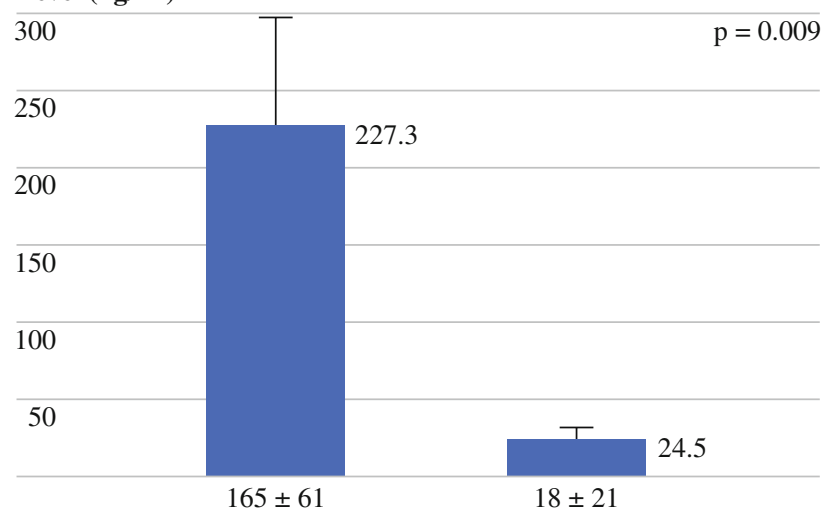

b
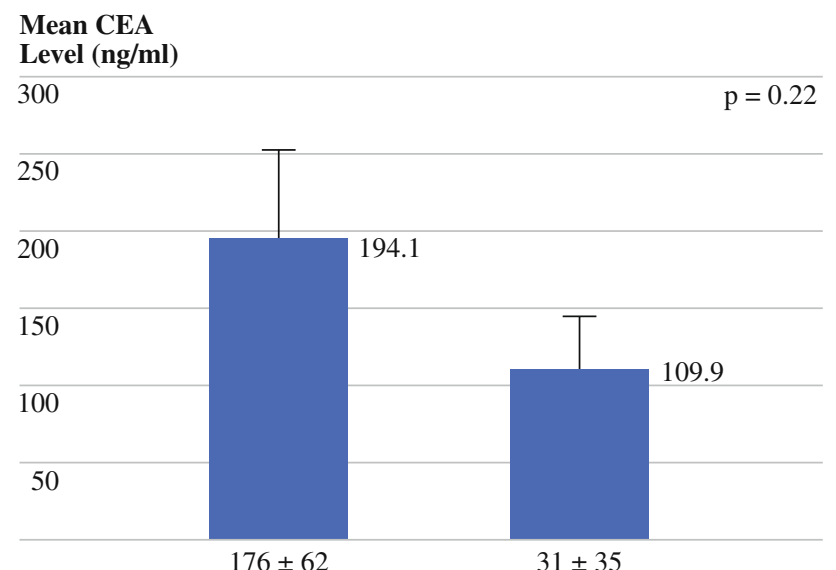

\section{$176 \pm 62$ \\ Mean Period before Hepatectomy (Days)}

c
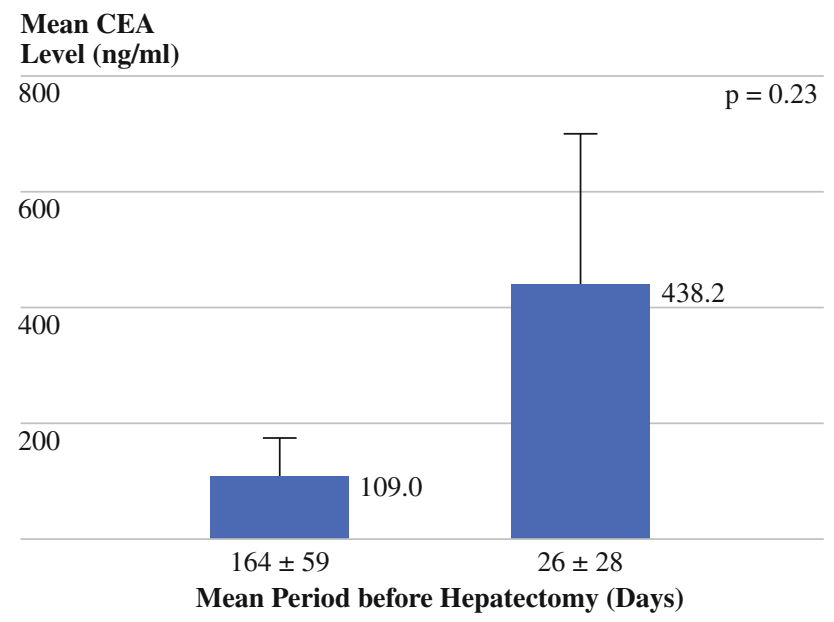

FIG. 2 Change of mean CEA level measured before and after last preoperative chemotherapy line in patients in whom radiological a response $(N=68)$, b stabilization $(N=26)$, or c progression $(N=19)$ of colorectal liver metastases was observed

Although not statistically different, observed changes in mean CA19.9 levels measured before and after preoperative chemotherapy were in accordance with their
TABLE 3 Change of preoperative tumor marker levels (cutoff 20\%) compared with clinical response at CT imaging after chemotherapy

\begin{tabular}{lllll}
\hline \multicolumn{5}{c}{${\text { Clinical response at } \mathrm{CT}^{\mathrm{b}}}$} \\
\cline { 2 - 5 } & Response & Stabilization & Progression & Total \\
\hline Change from preoperative CEA level ${ }^{\mathrm{a}}$ & & \\
Response & 61 & 13 & 1 & $75(66 \%)$ \\
Stabilization & 3 & 11 & 0 & $14(12 \%)$ \\
Progression & 4 & 2 & 18 & $24(21 \%)$ \\
Total & $68(60 \%)$ & $26(23 \%)$ & $19(17 \%)$ & $113(100 \%)$ \\
Change from preoperative CA19.9 level & & \\
Response & 38 & 9 & 1 & $48(70 \%)$ \\
Stabilization & 1 & 4 & 3 & $8(12 \%)$ \\
Progression & 0 & 5 & 7 & $12(18 \%)$ \\
Total & $39(57 \%)$ & $18(26 \%)$ & $11(16 \%)$ & $68(100 \%)$ \\
\hline
\end{tabular}

$C T$ computed tomography, $C E A$ carcinoembryonic antigen, $C A$ carbohydrate antigen

${ }^{\text {a }}$ Cutoff point $20 \%$

b According to RECIST criteria $^{22}$

corresponding radiological response categories, as demonstrated in Fig. 3a-c. Biological (CA19.9) and radiological response correlated favorably in $72 \%$ of cases (49/ 68), with a kappa value of 0.46 (95\% CI: 0.25-0.59) (Table 3). Sensitivity was 91\% (95\% CI: 84-99\%), specificity was 64\% (95\% CI: 35-92\%), PPV was 93\% (95\% CI: $86-100 \%)$, and NPV was $58 \%$ (95\% CI: $30-86 \%)$.

\section{Hepatic Resection}

To increase the volume of future remnant liver, preoperative portal vein embolization was performed in 14 patients $(12 \%)$. Major hepatectomy was performed in 62 patients $(52 \%)$, and any form of intraoperative vascular occlusion was needed in 96 patients (81\%). Hepatic resection was both macroscopically and microscopically complete in 71 patients $(60 \%)$ (Table 2). One patient (1\%) died at the $19^{\text {th }}$ postoperative day due to septic shock with multi-organ failure. Postoperative morbidity was 36\% (51 complications: Clavien grade I/II, $N=42$; grade III/IV, $N=9) .{ }^{27}$ Adjuvant chemotherapy was administered in the majority $(88 \%)$ of patients.

\section{Long-Term Outcome}

After a mean follow-up of 34 months (45 months for surviving patients), 20 patients (17\%) were alive and disease free, $18(15 \%)$ were alive with disease recurrence, and $81(68 \%)$ had died. Disease recurrence was diagnosed in 99 patients $(83 \%)$. Repeat hepatectomy for intrahepatic recurrence was performed in $42 \%$ of patients, and 26 patients $(22 \%)$ underwent resection of an extrahepatic recurrence. 


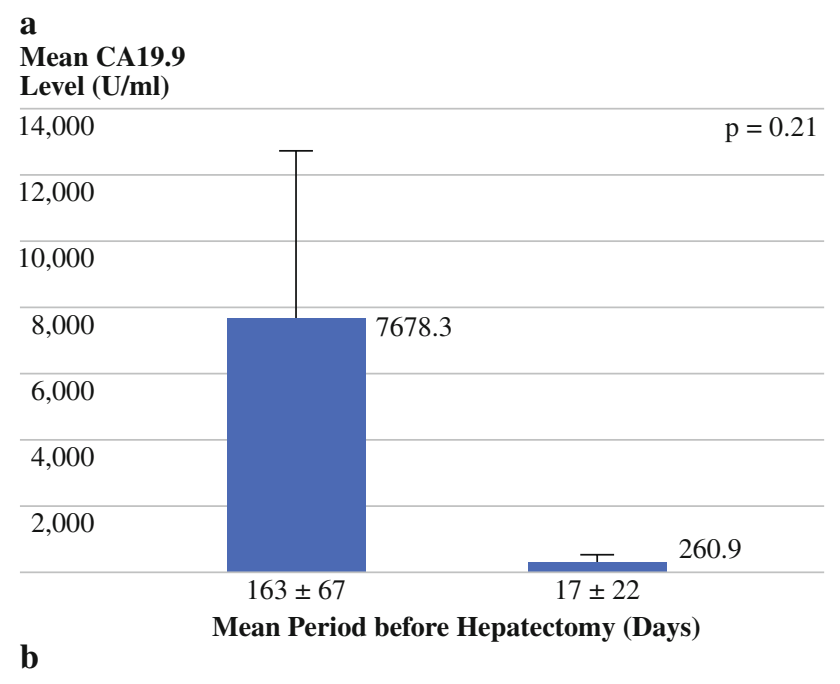

Mean CA19.9

Level $(\mathrm{U} / \mathrm{ml})$

$600 \quad \mathrm{p}=0.86$

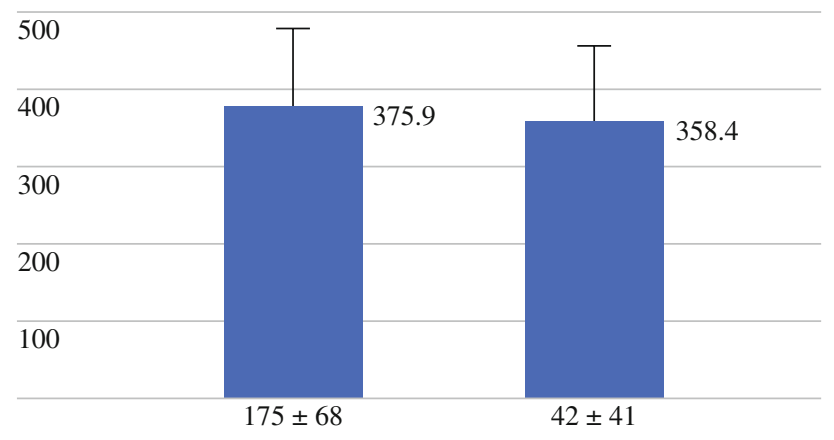

c

Mean Period before Hepatectomy (Days)

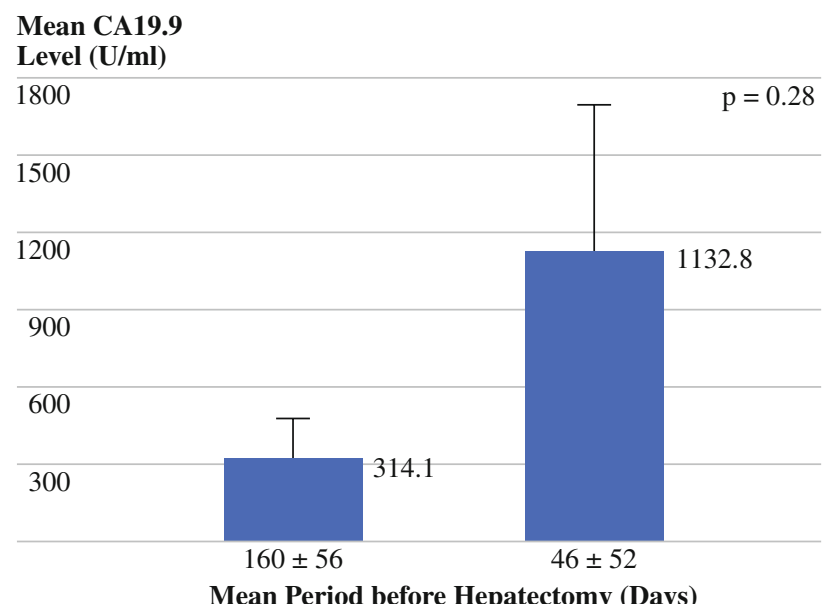

FIG. 3 Change of mean CA19.9 level measured before and after last preoperative chemotherapy line in patients in whom radiological. a response $(N=39)$, b stabilization $(N=18)$, or c progression $(N=11)$ of colorectal liver metastases was observed

Overall Survival (OS) Considering the impact of CEA evolution on OS, 3- and 5-year OS were $44 \%$ and $28 \%$ if patients showed a response of CEA level after chemotherapy, $37 \%$ and $28 \%$ in case of stabilization, and $41 \%$ and $14 \%$ if CEA level had increased more than $20 \%$, respectively $(P=0.65) \quad$ (Fig. 4a). Median OS was 33 months in each group.

For CA19.9, 3- and 5-year OS were 55\% and 36\% in case of response, $40 \%$ and $0 \%$ when stabilized, and $0 \%$ and $0 \%$ when CA19.9 level had increased more than $20 \%$ $(P=0.003)$ (Fig. 4b). Median OS was 40 months in the response group, 31 months in the stabilization group, and 22 months in case of progression of CA19.9 levels.

When CLM showed a radiological response following preoperative chemotherapy, 3- and 5-year OS were $42 \%$ and $29 \%$, respectively, compared with $46 \%$ and $28 \%$ if lesions were stabilized, and $36 \%$ and $7 \%$ if progression of CLM at CT imaging was observed $(P=0.23$; median OS of 34,32 , and 30 months, respectively) (Fig. 4c).

Progression-Free Survival (PFS) Median PFS was 9 months if CEA level responded after chemotherapy, compared with 7 months in both stabilization and progression groups $(P=0.17)$ (Fig. $4 \mathrm{~d})$.

Progression-free survival was significantly influenced by CA19.9 evolution, as none of the progressing patients was recurrence free after 3 years, compared with $16 \%$ and $14 \%$ of patients whose CA19.9 level showed response or stabilization, respectively $(P=0.04$; median $\mathrm{PFS}$ was 10 months in the response group versus 7 months for both stabilization and progression groups) (Fig. 4e).

Finally, median PFS in case of radiological progression tended to be lower than that observed after radiological response or stabilization ( 7 months versus 9 months and 9 months, respectively; $P=0.06$ ) (Fig. 4f).

\section{Prognostic Factors of Overall Survival}

On univariate analysis, study variables that were associated with poor OS were synchronous CLM, $\geq 4$ CLM at diagnosis, diameter of largest metastasis $\geq 35 \mathrm{~mm}$, bilateral CLM, initial unresectability, and progression of CA19.9 level after preoperative chemotherapy (Table 4).

After introducing all factors with univariate $P \leq 0.10$ in a Cox proportional hazard model, three factors were identified as independent predictors of poor OS: $\geq 4$ CLM at diagnosis, largest metastasis diameter $\geq 35 \mathrm{~mm}$, and radiological (but not biological) progression of CLM following preoperative chemotherapy (Table 4).

\section{Prognostic Factors of Progression-Free Survival}

On univariate analysis, nine factors were identified which significantly influenced PFS: female gender, synchronous CLM, $\geq 3$ CLM at diagnosis, bilateral CLM, type of last preoperative chemotherapy, radiological response 
a

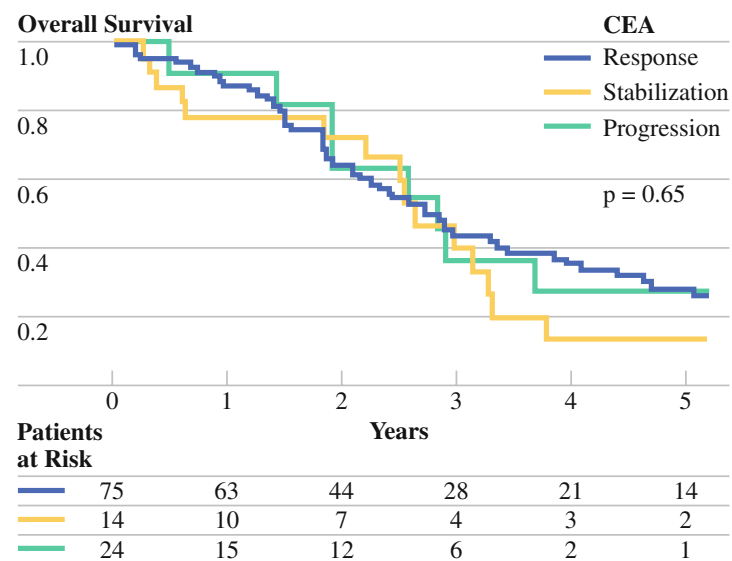

b

Overall Survival
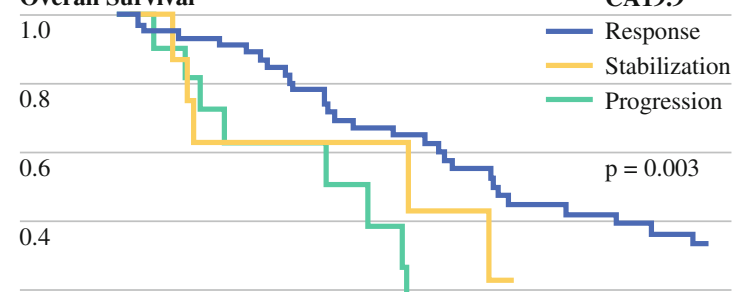

0.2

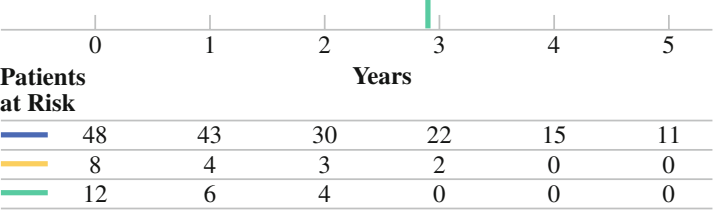

c

Overall Survival

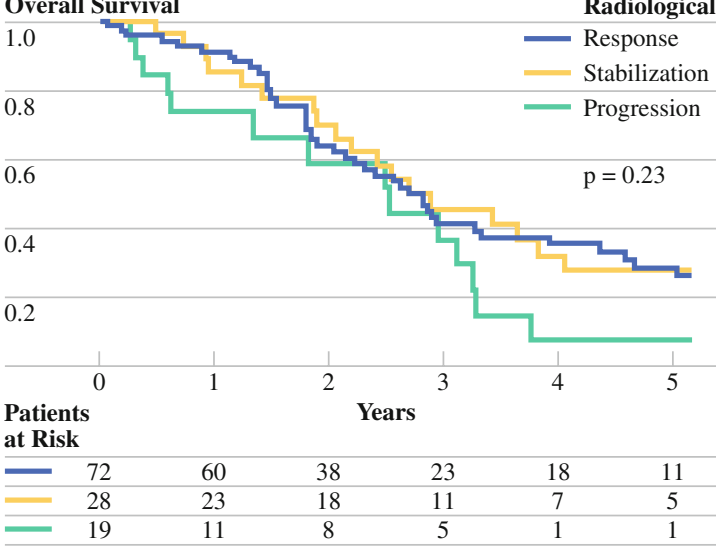

FIG. 4 Impact of change of CEA level (a and d) and CA19.9 level (b and e) and radiological response (c and f) after preoperative chemotherapy on overall survival $(\mathbf{a}-\mathbf{c})$ and progression-free survival

following chemotherapy, biological response of CA19.9 level after chemotherapy, administration of adjuvant chemotherapy, and surgical margin status after hepatectomy (Table 5).

\section{d}

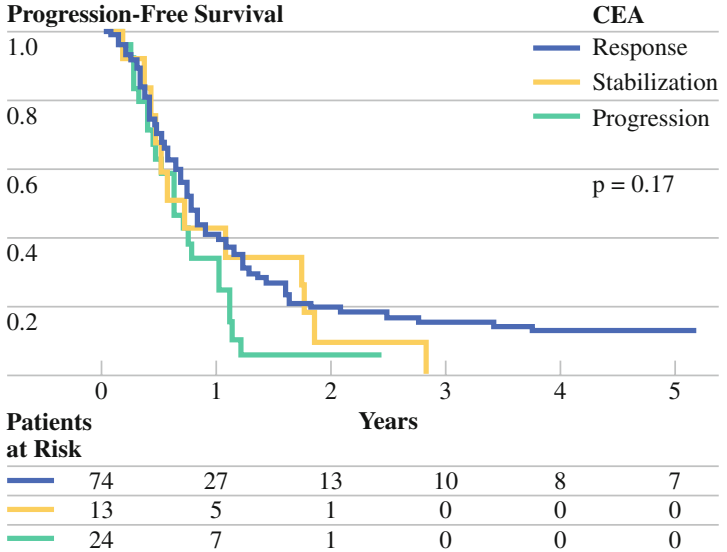

e

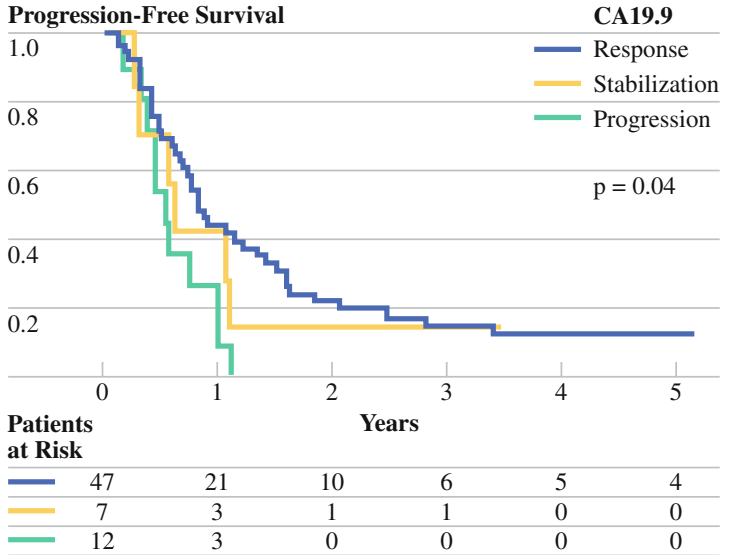

f

Progression-Free Survival

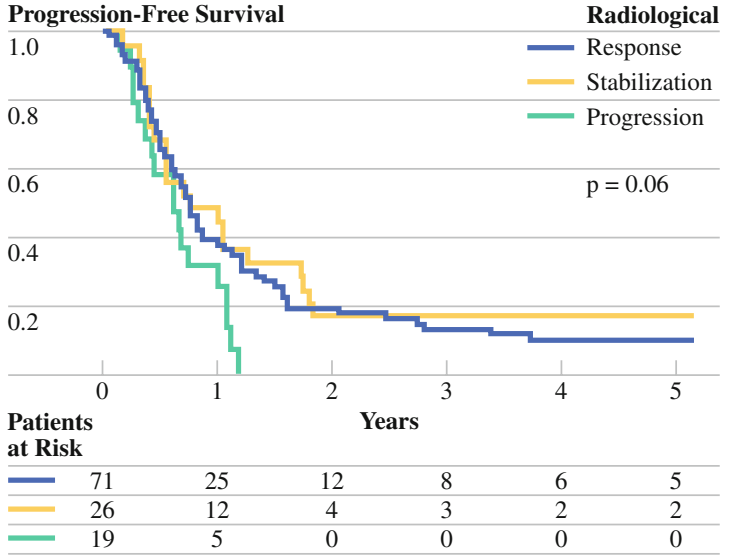

(d-f) (a cutoff point of 20\% was used to define biological response, stabilization, and progression; radiological response was determined according to RECIST criteria $^{22}$ )

On multivariate analysis, female gender, $\geq 3$ CLM at diagnosis, and progression of CA19.9 level after chemotherapy, but not radiological progression, were independent predictors of decreased PFS (Table 5). 
TABLE 4 Univariate and multivariate analysis of overall survival

\begin{tabular}{|c|c|c|c|c|c|}
\hline Variable & $N$ & 5-Year OS (\%) & UV $P$ & $\mathrm{MV}^{\mathrm{a}} P$ & $\operatorname{HR}(95 \% \mathrm{CI})$ \\
\hline All patients & 119 & 26 & & & \\
\hline \multicolumn{6}{|l|}{ Patient factors } \\
\hline \multicolumn{6}{|l|}{ Gender } \\
\hline Male & 79 & 23 & 0.12 & - & - \\
\hline Female & 40 & 30 & & & \\
\hline \multicolumn{6}{|l|}{ Age at hepatectomy } \\
\hline$<60$ years & 46 & 21 & 0.49 & - & - \\
\hline$\geq 60$ years & 73 & 29 & & & \\
\hline \multicolumn{6}{|l|}{ Primary malignancy } \\
\hline \multicolumn{6}{|l|}{ Location } \\
\hline Colon & 95 & 25 & 0.73 & - & - \\
\hline Rectum & 24 & 29 & & & \\
\hline \multicolumn{6}{|l|}{$\mathrm{T}$ classification } \\
\hline $1-2$ & 9 & 22 & 0.24 & - & - \\
\hline $3-4$ & 74 & 24 & & & \\
\hline \multicolumn{6}{|l|}{$\mathrm{N}$ classification } \\
\hline 0 & 33 & 25 & 0.77 & - & - \\
\hline $1-2$ & 52 & 18 & & & \\
\hline \multicolumn{6}{|l|}{ CLM at diagnosis } \\
\hline \multicolumn{6}{|l|}{ Timing of diagnosis ${ }^{\mathrm{b}}$} \\
\hline Synchronous & 41 & 14 & 0.009 & NS & - \\
\hline Metachronous & 78 & 33 & & & \\
\hline \multicolumn{6}{|l|}{ No. of CLM } \\
\hline$<4$ & 78 & 32 & 0.002 & $<0.001$ & $2.6(1.6-4.3)$ \\
\hline$\geq 4$ & 37 & 17 & & & \\
\hline \multicolumn{6}{|l|}{ Max. size of CLM } \\
\hline$<35 \mathrm{~mm}$ & 50 & 34 & 0.005 & 0.002 & $2.2(1.3-3.7)$ \\
\hline$\geq 35 \mathrm{~mm}$ & 58 & 16 & & & \\
\hline \multicolumn{6}{|l|}{ Distribution } \\
\hline Unilateral & 61 & 35 & 0.004 & NS & - \\
\hline Bilateral & 57 & 16 & & & \\
\hline \multicolumn{6}{|l|}{ Initial resectability } \\
\hline Yes & 64 & 40 & 0.01 & NS & - \\
\hline No & 55 & 15 & & & \\
\hline \multicolumn{6}{|l|}{ Hepatic resection } \\
\hline \multicolumn{6}{|c|}{ Preoperative chemotherapy } \\
\hline \multicolumn{6}{|c|}{ Total no. of lines } \\
\hline 1 & 87 & 30 & 0.6 & - & - \\
\hline$\geq 2$ & 32 & 13 & & & \\
\hline \multicolumn{6}{|l|}{ Total no. of cycles } \\
\hline$<10$ & 72 & 34 & 0.13 & - & - \\
\hline$\geq 10$ & 40 & 7 & & & \\
\hline \multicolumn{6}{|c|}{ Regimen last preoperative line } \\
\hline 5-FU LV & 32 & 25 & 0.17 & - & - \\
\hline 5-FU LV oxaliplatin & 61 & 32 & & & \\
\hline 5-FU LV irinotecan & 18 & 16 & & & \\
\hline Other & 8 & 0 & & & \\
\hline
\end{tabular}


TABLE 4 continued

\begin{tabular}{|c|c|c|c|c|c|}
\hline Variable & $N$ & 5 -Year OS $(\%)$ & UV $P$ & $\mathrm{MV}^{\mathrm{a}} P$ & $\mathrm{HR}(95 \% \mathrm{CI})$ \\
\hline \multicolumn{6}{|l|}{ Chronomodulated therapy } \\
\hline Yes & 59 & 28 & 0.57 & - & - \\
\hline No & 60 & 23 & & & \\
\hline \multicolumn{6}{|c|}{ Radiological response at CT $\operatorname{scan}^{\mathrm{c}}$} \\
\hline Response or stabilization & 100 & 29 & 0.09 & 0.004 & $2.6(1.3-4.9)$ \\
\hline Progression & 19 & 7 & & & \\
\hline \multicolumn{6}{|l|}{ Biological response, CEA $^{\mathrm{d}}$} \\
\hline Response or stabilization & 89 & 28 & 0.36 & - & - \\
\hline Progression & 24 & 14 & & & \\
\hline \multicolumn{6}{|l|}{ Biological response, CA19.9 ${ }^{\mathrm{d}}$} \\
\hline Response or stabilization & 56 & 33 & 0.002 & NS & - \\
\hline Progression & 12 & 0 & & & \\
\hline \multicolumn{6}{|l|}{ Extent of hepatic resection } \\
\hline Minor ( $<3$ segments $)$ & 57 & 32 & 0.06 & NS & - \\
\hline Major ( $\geq 3$ segments) & 62 & 20 & & & \\
\hline \multicolumn{6}{|l|}{ Resection type } \\
\hline Anatomical & 58 & 31 & 0.49 & - & - \\
\hline Nonanatomical & 26 & 29 & & & \\
\hline Both & 35 & 12 & & & \\
\hline \multicolumn{6}{|l|}{ Vascular occlusion } \\
\hline Yes & 97 & 25 & 0.95 & - & - \\
\hline No & 17 & 30 & & & \\
\hline \multicolumn{6}{|c|}{ Intraoperative $\mathrm{RBC}$ transfusion } \\
\hline Yes & 32 & 25 & 0.85 & - & - \\
\hline No & 60 & 30 & & & \\
\hline \multicolumn{6}{|l|}{ Postoperative chemotherapy } \\
\hline Yes & 105 & 27 & 0.37 & - & - \\
\hline No & 11 & 12 & & & \\
\hline \multicolumn{6}{|l|}{ Surgical margin status ${ }^{\mathrm{e}}$} \\
\hline R0 & 71 & 28 & 0.16 & - & - \\
\hline $\mathrm{R} 1$ & 43 & 21 & & & \\
\hline $\mathrm{R} 2$ & 4 & 25 & & & \\
\hline
\end{tabular}

$O S$ overall survival, $U V$ univariate, $M V$ multivariate, $H R$ hazard ratio, $C I$ confidence interval, $C L M$ colorectal liver metastases, $N S$ not significant, 5-FU 5-fluorouracil, $L V$ leucovorin, $C T$ computed tomography, $C E A$ carcinoembryonic antigen, $C A$ carbohydrate antigen, $R B C$ red blood cell

a Variables with univariate $P \leq 0.10$ were entered in the Cox regression model

${ }^{\mathrm{b}}$ Synchronous = diagnosed before, during, or within 3 months after colorectal resection

c According to RECIST criteria ${ }^{22}$

${ }^{\mathrm{d}}$ Cutoff point $20 \%$

e R0: complete surgical resection with a negative surgical margin at histopathology; R1: invaded surgical margins according to the pathologist; R2: macroscopic tumor remnant intraoperatively

\section{Discrepancies Between Biological and Radiological Evolution}

Of patients in whom CEA level had increased more than $20 \%$ during chemotherapy treatment $(N=24)$, response or stabilization was observed at CT imaging in six patients. In addition, of 89 patients whose CEA level showed response or stabilization during chemotherapy, one had progression of disease at CT imaging. All of these patients developed disease recurrence shortly after hepatic resection (median 7 months), and three of them had died at last follow-up.

When progression of CA19.9 levels was observed during chemotherapy $(N=12)$, five patients had radiological response or stabilization. Furthermore, of 56 patients with 
TABLE 5 Univariate and multivariate analysis of progression-free survival

\begin{tabular}{|c|c|c|c|c|c|}
\hline Variable & $N$ & 5-Year PFS (\%) & UV $P$ & $\mathrm{MV}^{\mathrm{a}} P$ & $\mathrm{HR}(95 \% \mathrm{CI})$ \\
\hline All patients & 116 & 9 & & & \\
\hline \multicolumn{6}{|l|}{ Patient factors } \\
\hline \multicolumn{6}{|l|}{ Gender } \\
\hline Male & 76 & 20 & 0.05 & 0.04 & $1.9(1.0-3.5)$ \\
\hline Female & 40 & 3 & & & \\
\hline \multicolumn{6}{|l|}{ Age at hepatectomy } \\
\hline$<70$ years & 92 & 9 & 0.85 & - & - \\
\hline$\geq 70$ years & 24 & 10 & & & \\
\hline \multicolumn{6}{|l|}{ Primary malignancy } \\
\hline \multicolumn{6}{|l|}{ Location } \\
\hline Colon & 93 & 9 & 0.44 & - & - \\
\hline Rectum & 23 & 10 & & & \\
\hline \multicolumn{6}{|l|}{$\mathrm{T}$ classification } \\
\hline $1-2$ & 9 & 0 & 0.64 & - & - \\
\hline $3-4$ & 72 & 9 & & & \\
\hline \multicolumn{6}{|l|}{$\mathrm{N}$ classification } \\
\hline 0 & 32 & 8 & 0.91 & - & - \\
\hline $1-2$ & 51 & 5 & & & \\
\hline \multicolumn{6}{|l|}{ CLM at diagnosis } \\
\hline \multicolumn{6}{|l|}{ Timing of diagnosis ${ }^{\mathrm{b}}$} \\
\hline Synchronous & 39 & 3 & 0.05 & NS & - \\
\hline Metachronous & 77 & 13 & & & \\
\hline \multicolumn{6}{|l|}{ No. of CLM } \\
\hline$<3$ & 62 & 18 & $<0.001$ & 0.02 & $1.9(1.1-3.4)$ \\
\hline$\geq 3$ & 50 & 0 & & & \\
\hline \multicolumn{6}{|l|}{ Max. size of CLM } \\
\hline$<35 \mathrm{~mm}$ & 48 & 15 & 0.28 & - & - \\
\hline$\geq 35 \mathrm{~mm}$ & 57 & 6 & & & \\
\hline \multicolumn{6}{|l|}{ Distribution } \\
\hline Unilateral & 58 & 18 & 0.006 & NS & - \\
\hline Bilateral & 57 & 0 & & & \\
\hline \multicolumn{6}{|l|}{ Initial resectability } \\
\hline Yes & 63 & 18 & 0.28 & - & - \\
\hline No & 53 & 3 & & & \\
\hline \multicolumn{6}{|l|}{ Hepatic resection } \\
\hline \multicolumn{6}{|c|}{ Preoperative chemotherapy } \\
\hline \multicolumn{6}{|c|}{ Total no. of lines } \\
\hline 1 & 86 & 9 & 0.97 & - & - \\
\hline$\geq 2$ & 30 & 10 & & & \\
\hline \multicolumn{6}{|l|}{ Total no. of cycles } \\
\hline$<10$ & 69 & 11 & 0.08 & NS & - \\
\hline$\geq 10$ & 40 & 4 & & & \\
\hline \multicolumn{6}{|c|}{ Regimen last preoperative line } \\
\hline 5-FU LV & 31 & 10 & $\mathbf{0 . 0 3}$ & NS & - \\
\hline 5-FU LV oxaliplatin & 61 & 12 & & & \\
\hline 5-FU LV irinotecan & 16 & 6 & & & \\
\hline Other & 8 & 0 & & & \\
\hline
\end{tabular}


TABLE 5 continued

\begin{tabular}{|c|c|c|c|c|c|}
\hline Variable & $N$ & 5-Year PFS $(\%)$ & UV $P$ & $\mathrm{MV}^{\mathrm{a}} P$ & $\operatorname{HR}(95 \% \mathrm{CI})$ \\
\hline \multicolumn{6}{|l|}{ Chronomodulated therapy } \\
\hline Yes & 59 & 9 & 0.89 & - & - \\
\hline No & 57 & 10 & & & \\
\hline \multicolumn{6}{|c|}{ Radiological response at CT $\operatorname{scan}^{\mathrm{c}}$} \\
\hline Response or stabilization & 97 & 11 & 0.02 & NS & - \\
\hline Progression & 19 & 0 & & & \\
\hline \multicolumn{6}{|l|}{ Biological response, CEA $^{\mathrm{d}}$} \\
\hline Response or stabilization & 87 & 10 & 0.07 & NS & - \\
\hline Progression & 24 & 0 & & & \\
\hline \multicolumn{6}{|l|}{ Biological response, CA19.9 ${ }^{\mathrm{d}}$} \\
\hline Response or stabilization & 54 & 13 & 0.01 & 0.002 & $3.2(1.6-6.6)$ \\
\hline Progression & 12 & 0 & & & \\
\hline \multicolumn{6}{|l|}{ Extent of hepatic resection } \\
\hline Minor $(<3$ segments $)$ & 54 & 13 & 0.31 & - & - \\
\hline Major ( $\geq 3$ segments) & 62 & 6 & & & \\
\hline \multicolumn{6}{|l|}{ Resection type } \\
\hline Anatomical & 57 & 14 & 0.12 & - & - \\
\hline Nonanatomical & 24 & 9 & & & \\
\hline Both & 35 & 0 & & & \\
\hline \multicolumn{6}{|l|}{ Vascular occlusion } \\
\hline Yes & 95 & 8 & 0.49 & - & - \\
\hline No & 17 & 18 & & & \\
\hline \multicolumn{6}{|c|}{ Intraoperative $\mathrm{RBC}$ transfusion } \\
\hline Yes & 32 & 6 & 0.90 & - & - \\
\hline No & 58 & 13 & & & \\
\hline \multicolumn{6}{|l|}{ Postoperative chemotherapy } \\
\hline Yes & 105 & 10 & 0.009 & NS & - \\
\hline No & 10 & 0 & & & \\
\hline \multicolumn{6}{|l|}{ Surgical margin status ${ }^{\mathrm{e}}$} \\
\hline R0 & 69 & 13 & 0.04 & NS & - \\
\hline $\mathrm{R} 1$ & 42 & 3 & & & \\
\hline $\mathrm{R} 2$ & 4 & 0 & & & \\
\hline
\end{tabular}

PFS progression-free survival, $U V$ univariate, $M V$ multivariate, $H R$ hazard ratio, $C I$ confidence interval, $C L M$ colorectal liver metastases, $N S$ not significant, 5-FU 5-fluorouracil, $L V$ leucovorin, $C T$ computed tomography, $C E A$ carcinoembryonic antigen, $C A$ carbohydrate antigen, $R B C$ red blood cell

${ }^{a}$ Variables with univariate $P \leq 0.10$ were entered in the Cox regression model

b Synchronous = diagnosed before, during, or within 3 months after colorectal resection

c According to RECIST criteria $^{22}$

${ }^{\mathrm{d}}$ Cutoff point $20 \%$

${ }^{\mathrm{e}}$ R0: complete surgical resection with a negative surgical margin at histopathology; R1: invaded surgical margins according to the pathologist; R2: macroscopic tumor remnant intraoperatively

response or stabilization of CA19.9 level during chemotherapy, 4 had radiological progression of disease. Again, all of these patients recurred shortly after hepatic resection (median 6 months), and at last follow-up seven had died of disease progression.

\section{DISCUSSION}

As the decisional value of tumor marker evolution during chemotherapy in patients scheduled for hepatic resection of CLM is still unclear, we aimed to define the 
correlation between biological and radiological response following preoperative chemotherapy as well as their impact on long-term outcome in this particular patient group.

In the present study, CEA evolution was found to be highly correlated with radiological response after preoperative chemotherapy, with sensitivity and specificity rates exceeding $90 \%$. However, while radiological progression independently predicted decreased OS, change in CEA level after chemotherapy did not significantly impact longterm outcome, although a trend for decreased survival was observed in patients with increased CEA level (5-year OS $14 \%$ versus $28 \%$ ).

Sensitivity of CA19.9 evolution was comparable to that observed for CEA, with however, a lower specificity. In contrast to CEA evolution, progression of CA19.9 level during chemotherapy had negative impact on both OS and PFS. In addition, progression of CA19.9 level after chemotherapy, but not radiological response, was an independent predictor of poor PFS.

In several large patient series, various cutoff points of elevated tumor marker levels before hepatectomy for CLM have been related to worse long-term outcome. ${ }^{13-17}$ However, little evidence exists regarding the decisional value of biological response during chemotherapy. In only four publications was the correlation between biological and radiological response analyzed. ${ }^{18-20,28}$ Ward et al. assessed the accuracy of tumor marker evolution (CEA, CA-195, and CA-242) in monitoring patients treated by chemotherapy for advanced colorectal cancer. In that study, CEA was found to have the best predictive value in monitoring disease course during chemotherapy, which correlated favorably with radiological changes in $88 \%$ of patients. ${ }^{20}$ More recently, Wang et al. reported a lower rate of agreement between imaging studies and change in CEA level $(68 \%) .{ }^{19}$ In 2006, Boppudi et al. concluded that there existed a major lack of agreement between tumoral changes at CT imaging and changes in CEA level in patients treated by selective internal radiation therapy (SIRT) for unresectable CLM. $^{28}$ Finally, Iwanicki-Caron et al. recently reported that CEA kinetics is an accurate method to identify disease progression in patients with advanced colorectal cancer. ${ }^{18}$ Importantly, these studies included only patients treated in a nonsurgical setting, with colorectal metastases not only confined to the liver, normal baseline CEA levels in some of them, thereby hampering data interpretation, and no evaluation of CA19.9 kinetics. As the value of CEA and CA19.9 evolution still remains a matter of debate, we decided to define both the correlation between biological and radiological response on chemotherapy and their comparative impact on long-term outcome, in a well-defined population of patients with isolated CLM who were all treated by chemotherapy followed by hepatic resection and all of whom had elevated baseline tumor marker levels. In this way, it was logical to assume that tumor marker levels were only related to liver metastases, thereby enabling us to draw reliable conclusions. In addition, as more than two-thirds of our patients were treated by modern chemotherapy regimens, and by using the RECIST criteria, our results reflect current standard of care. However, although strict inclusion criteria were used, some variation exists in the severity of metastatic disease and in the type of chemotherapy used within this "surgical" population.

When using a cutoff point of $20 \%$ change to define biological response after chemotherapy, biological response correlated favorably with radiological response in $80 \%$ and $72 \%$ of patients, with kappa values of 0.62 and 0.46, when either CEA or CA19.9 was analyzed, respectively. It was noteworthy that patients in whom discrepancy existed between radiological and biological response all recurred shortly after hepatectomy, and most of them had died at last follow-up. Therefore, determining biological response after chemotherapy provides important additional information, as patients whose metastases did not progress radiologically but who did demonstrate biological progression had similar prognosis compared with patients whose metastases showed radiological progression.

Concerning the correlation between biological and radiological response to chemotherapy, an important question that arises is whether tumor marker evolution should replace radiological evaluation. In patients with potentially resectable CLM, CT imaging remains the gold standard in evaluating chemotherapy results, as it enables evaluation of technical resectability of CLM. However, radiological evaluation should be combined in these patients with biological response, as this most accurately evaluates chemotherapy response and thus provides the best information regarding long-term outcome. Although we have studied a "surgical" population, almost half of the patients (46\%) were initially unresectable, and might not have been treated surgically in less specialized centers. Therefore, our results concerning biological and radiological correlation might also be applicable for a nonsurgical population. Thus, the efficacy of systemic chemotherapy administered in a nonsurgical treatment setting can be adequately monitored by determining biological response, thereby lowering the need for imaging studies. In this way, a reduction in health care costs could be achieved, combined with an improvement in patient comfort. Whether this hypothesis is true or not should be confirmed in future studies.

In our study, CEA evolution did not significantly influence OS and PFS, which is in contrast with the results reported by Iwanicki-Caron et al., Wang et al. and Boppudi et al., since in these series decrease in CEA level was significantly related to better survival. ${ }^{18,19,28}$ This could be 
related to the fact that, in some of the cited studies, patients with normal baseline tumor marker levels were also analyzed. In contrast, in our series, progression of CA19.9 level significantly influenced OS, and more importantly, it independently predicted poor PFS, thereby emphasizing its importance in estimating individual outcome. The value of CA19.9 evolution in patients with CLM treated by preoperative chemotherapy has to our knowledge never been explored, and it is noteworthy that it could be more accurate than CEA. Radiological progression of CLM following preoperative chemotherapy was found to be an independent predictor of poor OS, which is in accordance with our previous publication reporting a similar relationship between radiological progression and long-term outcome. ${ }^{12}$

In summary, the results of this study clearly show that, in patients with CLM treated by systemic chemotherapy, biological response (as measured by both CEA and CA19.9) and radiological response are closely correlated. Furthermore, CA19.9 evolution is an even better prognostic tool than radiological response with regards to PFS. In patients treated by systemic chemotherapy in a nonsurgical treatment setting, biological response can decrease the need for imaging studies. In addition, in patients with potentially resectable CLM, although CT imaging remains irreplaceable to determine resectability, biological response can provide relevant complementary information concerning individual outcome. In the future, our results need to be confirmed by other studies.

OPEN ACCESS This article is distributed under the terms of the Creative Commons Attribution Noncommercial License which permits any noncommercial use, distribution, and reproduction in any medium, provided the original author(s) and source are credited.

\section{REFERENCES}

1. Scheele J, Altendorf-Hofmann A. Resection of colorectal liver metastases. Langenbecks Arch Surg. 1999;384:313-27.

2. Adam R. Chemotherapy and surgery: new perspectives on the treatment of unresectable liver metastases. Ann Oncol. 2003;14(Suppl 2):ii13-6.

3. Adam R, Avisar E, Ariche A, et al. Five-year survival following hepatic resection after neoadjuvant therapy for nonresectable colorectal. Ann Surg Oncol. 2001;8:347-53.

4. Adam R, Aloia T, Lévi F, et al. Hepatic resection after rescue cetuximab treatment for colorectal liver metastases previously refractory to conventional systemic therapy. J Clin Oncol. 2007;25:4593-602.

5. Alberts SR, Horvath WL, Sternfeld WC, et al. Oxaliplatin, fluorouracil, and leucovorin for patients with unresectable liver-only metastases from colorectal cancer: a North Central Cancer Treatment Group phase II study. J Clin Oncol. 2005;23:9243-9.

6. de la Camara J, Rodriguez J, Rotellar F, et al. Triplet therapy with oxaliplatin, irinotecan, 5-fluorouracil and folinic acid within a combined modality approach in patients with liver metastases from colorectal cancer. Proc Am Soc Clin Oncol. 2004;23:Abstr 3593.
7. Masi G, Cupini S, Marcucci L, et al. Treatment with 5-fluorouracil/folinic acid, oxaliplatin, and irinotecan enables surgical resection of metastases in patients with initially unresectable metastatic colorectal cancer. Ann Surg Oncol. 2006;13:58-65.

8. Pozzo C, Basso M, Cassano A, et al. Neoadjuvant treatment of unresectable liver disease with irinotecan and 5-fluorouracil plus folinic acid in colorectal cancer patients. Ann Oncol. 2004; 15:933-9.

9. Quenet F, Nordlinger B, Rivoire M, et al. Resection of previously unresectable liver metastases from colorectal cancer (LMCRC) after chemotherapy (CT) with CPT-11/L-OHP/LV5FU (Folfirinox): a prospective phase II trial. Proc Am Soc Clin Oncol. 2004;23:3613.

10. Allen PJ, Kemeny N, Jarnagin W, DeMatteo R, Blumgart L, Fong Y. Importance of response to neoadjuvant chemotherapy in patients undergoing resection of synchronous colorectal liver metastases. J Gastrointest Surg. 2003;7:109-15.

11. Nordlinger B, Sorbye H, Glimelius B, et al. Perioperative chemotherapy with FOLFOX4 and surgery versus surgery alone for resectable liver metastases from colorectal cancer (EORTC Intergroup trial 40983): a randomised controlled trial. Lancet. 2008;371:1007-16.

12. Adam R, Pascal G, Castaing D, et al. Tumor progression while on chemotherapy: a contraindication to liver resection for multiple colorectal metastases? Ann Surg. 2004;240:1052-61.

13. Adam R, Delvart V, Pascal G, et al. Rescue surgery for unresectable colorectal liver metastases downstaged by chemotherapy: a model to predict long-term survival. Ann Surg. 2004;240:644-57.

14. Fong Y, Fortner J, Sun RL, Brennan MF, Blumgart LH. Clinical score for predicting recurrence after hepatic resection for metastatic colorectal cancer: analysis of 1001 consecutive cases. Ann Surg. 1999;230:309-18.

15. Minagawa M, Makuuchi M, Torzilli G, et al. Extension of the frontiers of surgical indications in the treatment of liver metastases from colorectal cancer: long-term results. Ann Surg. 2000;231:487-99.

16. Nordlinger B, Guiguet M, Vaillant JC, Balladur P, Boudjema K, Bachellier P, Jaeck D. Surgical resection of colorectal carcinoma metastases to the liver. A prognostic scoring system to improve case selection, based on 1568 patients. Association Française de Chirurgie. Cancer. 1996;77:1254-62.

17. Scheele J, Stangl R, Altendorf-Hofmann A, Paul M. Resection of colorectal liver metastases. World J Surg. 1995;19:59-71.

18. Iwanicki-Caron I, Di Fiore F, Roque I, et al. Usefulness of the serum carcinoembryonic antigen kinetic for chemotherapy monitoring in patients with unresectable metastasis of colorectal cancer. J Clin Oncol. 2008;26:3681-6.

19. Wang WS, Lin JK, Lin TC, et al. Carcinoembryonic antigen in monitoring of response to systemic chemotherapy in patients with metastatic colorectal cancer. Int J Colorectal Dis. 2001;16:96101.

20. Ward U, Primrose JN, Finan PJ, Perren TJ, Selby P, Purves DA, Cooper EH. The use of tumour markers CEA, CA-195 and CA-242 in evaluating the response to chemotherapy in patients with advanced colorectal cancer. Br J Cancer. 1993; 67:1132-5.

21. Locker GY, Hamilton S, Harris J, et al. ASCO 2006 update of recommendations for the use of tumor markers in gastrointestinal cancer. J Clin Oncol. 2006;24:5313-27.

22. Therasse P, Arbuck SG, Eisenhauer EA, et al. New guidelines to evaluate the response to treatment in solid tumors. European Organization for Research and Treatment of Cancer, National Cancer Institute of the United States, National Cancer Institute of Canada. J Natl Cancer Inst. 2000;92:205-16. 
23. Azoulay D, Castaing D, Smail A, et al. Resection of nonresectable liver metastases from colorectal cancer after percutaneous portal vein embolization. Ann Surg. 2000;231:480-6.

24. Bismuth H. Surgical anatomy and anatomical surgery of the liver. World J Surg. 1982;6:3-9.

25. Couinaud C. Le foie: études anatomiques et chirurgicales. Paris: Masson et Cie; 1957.

26. Adam R, Bismuth H, Castaing D, et al. Repeat hepatectomy for colorectal liver metastases. Ann Surg. 1997;225:51-60.
27. Dindo D, Demartines N, Clavien PA. Classification of surgical complications: a new proposal with evaluation in a cohort of 6336 patients and results of a survey. Ann Surg. 2004;240:20513.

28. Boppudi S, Wickremesekera SK, Nowitz M, Stubbs R. Evaluation of the role of CT in the assessment of response to selective internal radiation therapy in patients with colorectal liver metastases. Australas Radiol. 2006;50:570-7. 